\title{
Towards an Artificial Architecture: About Superintelligent Space Philip Morel
}

\author{
EZCT Architecture \& Design Research, 32 rue Saint-Antoine,75004 Paris \\ ezct.philippe.morel@gmail.com
}

'Just as there are odors that dogs can smell and we cannot, as well as sounds that dogs can hear and we cannot, so too there are wavelengths of light we cannot see and flavors we cannot taste. Why then, given our brains wired the way they are, does the remark, "Perhaps there are thoughts we cannot think," surprise you? Evolution, so far, may possibly have blocked us from being able to think in some directions; there could be unthinkable thoughts.' (Hamming, 1980).

'We have preconceptions about how an intelligent robot should look and act, and these can blind us to what is already happening around us. To demand that artificial intelligence be humanlike is the same flawed logic as demanding that artificial flying be birdlike, with flapping wings. Robots will think different. To see how far artificial intelligence has penetrated our lives, we need to shed the idea that they will be humanlike.' (Kelly, 2012).

In the essay The Doctors of Tomorrow Will Be Supercomputers, published online at futurism.com, Leary (2017) says doctors will be replaced by artificial intelligence-fed supercomputers. This is in line with many theorists and futurists including Kelly (2012) who, on a more "material" level, declared in Wired: 'Even those areas of medicine not defined by paperwork, such as surgery, are becoming increasingly robotic. The rote tasks of any information-intensive job can be automated. It doesn't matter if you are a doctor, lawyer, architect, reporter, or even programmer: The robot takeover will be epic. And it has already begun. '[Kelly,2013] For this last author, with whom I can only agree, if we are now at a 'point of inflection' in the use of robots, it is because they have become intelligent machines. Indeed, intelligence is the whole question...

Architecture has, just as science or comics, its own history of superlatives. Thanks to the eponymous exhibition of Pistoia, in 1966 a 'Superarchitecture' (Superarchitettura) was added to the supercomputers and superheroes, which according to its theorists was an architecture of superproduction, superconsumption, superincitation to consume, supermarket, superman and super gasoline'[Superarchitettura, 1966]. As everyone knows, this superarchitecture has become, following Superstudio and Archizoom, a paradigm common to many other avant-garde groups which, according to a vanguard definition as 'dictatorship of reality', have naturally developed an "architecture of reality". In fact, superarchitecture had incorporated the essential elements of the reality of an era, if not a computational environment just nascent or embryonic whose signs appeared in the abstraction of grids and series, usually produced by hand on typewriters, or in the coldness of otherwise narrative images. Computation appeared here and there but without the calculation being truly effective and in action; everything was as if it still belonged to reverie. There are some reasons for that. Firstly, the European industrial context of the time as well as the hyper-politicization of the social sphere obviously played against an in-depth analysis of computation as well as the various but inevitable consequences of the quantitative and qualitative evolution of computers. Among the architects and researchers who were most interested in this issue, very few had the opportunity for direct access to the machines themselves, which were expensive, cumbersome, and therefore extremely limited in Europe. Secondly, the visceral rejection of modernism contributed to a global discredit on a whole section of research inspired by science. "Proto-computational" architects and artists were often wrongly labeled as scientistic or naively progressive late modernist people, at the very moment when the concept of scientific progress was losing its authority. Like Leonardo Mosso in Italy, Seguí and 
Buenaventura $(1968,1974)$, active at the Computing Center of the University of Madrid (CCUM) - an experimental space developed and financed by IBM from 1968 -, Jozef Jankovič in Czechoslovakia or Konstantinos Doxiadis in Athens, many researchers working around the possibilities provided by computers appeared either as positivists, or as anti-Marxists, or both.

Finally, confronted with a formalism that rarely succeeded in renewing in depth the codes of modernism and with the voluntary or forced departure to the United States or South America of many emblematic figures of the European avant-gardes prior to 1939, it was more judicious to turn to truly new experiments like those of Pop Art or Conceptual Art than attempting to revive an outdated modernist art that became bourgeois and perfectly boring. Thanks to history of art and, to a lesser extent, to the history of architecture of the 1960s and 1970s, we know that the United States has been both more active and more receptive to experiments conducted with computers, whether on (programming) languages themselves or by making an instrumental use of these languages for the development of new forms and new spatial logics. The positive influence of great formalists of European origin such as Albers or Moholy-Nagy, many of whom had prestigious academic positions, the open minded attitude of the most original American creators (R. Buckminster Fuller, J. Cage, etc.), the relative absence of a monolithic stylistic school prior to the Second World War against which it would have been necessary to take an equally monolithic opposite stance, as well as the political and economic incentives for a new science and a new aesthetic have unambiguously allowed the emergence of research in the United States for which a context of refusal of ongoing scientific policies, then associated with liberalism and imperialism, did not seem the most favorable. In any case, and perhaps because in the 20th century the question of knowledge was mainly addressed by positivist philosophers (including Viennese neopositivists). Superarchitecture has never been theorized in relation to knowledge itself: neither as architecture of intelligence nor as architecture of superintelligence. Now, in the age of supercomputers and strong artificial intelligence, it is useful to define the latter, at least in its broad outlines.

To theorize the architecture of superintelligence or at least superintelligent space would require in all rigor to describe in advance the concept of superintelligence with its variants. We will assume here that the reader is already informed on this question and if it is not the case we invite him to have a look at the most influential books and papers (Bostrom, 2014). Nevertheless, as a very general reminder and before addressing the question of architecture, let us consider superintelligence as a radical increase in the general intelligence available, intelligence of machinic or human origin. If the hypothesis of the Singularity, as propounded by Kurzweil (2005), which we recall, is an empirical hypothesis based on historical facts, and is based on the exponential increase of machine intelligence, there is nothing in theory against the possibility of a parallel increase in biological intelligence, for example through genetic engineering. In fact, it is quite probable that the intelligence of the future will be integral and that it will not recognize the usual natural vs artificial and physical vs biological distinctions; the possibility of synthesizing DNA already demonstrates this. We are thus moving towards a distributed intelligence magma ("The" cloud...) which is to become the main component of any future architecture. An architecture massively generated by computational procedures - bringing together computation and data -, which will manifest itself through "superintelligent space". That this last one evokes various criteria, parameters and concepts associated with artificial intelligence - what exact type of machine learning, optimization of algorithm etc. - is not essential, moreover its artificiality is not as in the case of current AI a purely technical artificiality. Superintelligence is not the collective intelligence of neo-operatives and classical humanists, but not being human does not mean it is simply mechanical. It is in fact artificial, in every sense of that word. It refers to the general hybrid intelligence in circulation, human and artificial, whose effectiveness comes from the very possibility of making symbolical and numerical computation through a prior formalization, so thanks to its "artificialization". As a result of this general formalization and translation that flatten out the different forms of intelligence, it is an "artificial general intelligence" (AGI) that would have 
succeeded in integrating all the existing knowledge, giving birth to a concept of intelligence with both theoretical and practical efficiency, this further reinforcing the criterion of Turing universality while removing the anthropomorphism or biomimicry of the famous 'Turing test'.

Indeed, the objectives of this test first defined to "demonstrate" the possibility of an artificial intelligence at least equivalent to human intelligence, or more modestly to address criteria of intelligence, have been diverted to reintroduce a perfectly outdated anthropomorphism. In the field of architecture this anthropomorphism, indefensible and especially unusable, has been and is still regularly replaced by a biomimicry whose main asset, of a socio-political nature, is that it makes the extra-ordinary performance of computers and software ordinary and acceptable to the greatest number, including architects. It's not the least of the contradictions - I call it The Social Paradox of Artificial Intelligence - to see so many people, including those who need it, speak out against artificial intelligence and by extension against the general artificial intelligence, in favor of a respectable natural intelligence; natural but rather badly shared... Beyond the fact that the affirmation 'everything is in nature' makes us individuals of the Renaissance - so somewhat old ... - it does not say anything about the level of "naturalness" we are speaking about.

To say along with David Deutsch that all forms of intelligences, in vivo, in silico, etc., obey the same laws of physics (including those of quantum physics) and can therefore be reduced to an artificial general intelligence (AGI) whose real understanding still eludes us, is obviously not the same as finding that humans are experts in logic or chess and that ants are experts in swarm intelligence... all the more so when humans are surpassed at chess. The neo-romantic sanctification of nature as it manifests itself in the most obvious way also does not provide a solid argument for our inclination to regard ourselves as the alpha and omega of "Creation" and intelligence, and this only because so far we are the only species that made use of a rationalistic thought - in the service of a rational attitude and quite often also at the service of mass destruction. As K. Kelly[2012] reports it, 'we have preconceptions about how an intelligent robot should look and act, and these can blind us to what is already happening around us.' It was also as early as 1980, certainly concerning a different problem of epistemology, what was recalled by R. Hamming summoning our own sensory limitations, which should preserve us forever from any presumptuous attitude with regards to intelligence in general. In fact, neither with the most radical avant-gardes of the beginning of the $20^{\text {th }}$ century nor later with Peter Eisenman whose architecture is entirely directed to such an overtaking, did we really go beyond naturalism and mimesis. This for the simple reason that so far, we have never had to deal with an intelligence that, to take up the reactions to AlphaGo's victory against Lee Se-Dol from 9 to 15 March 2016, is external to us and manifests itself to us as an Alien. So far, the history of machines has merely been a story of artefacts understood as quantitative extensions of our intelligence, if not more simply mere extensions of our bodies. What a rocket engine and a microscope - including electronics - have in common is that they both incorporate our knowledge - our theories - for our own quantitative extension.

In one case we send our bodies, our tools, our theories or all together in orbit, in the other we project our concepts at the heart of matter to confront them with what we suppose to be ever finer degrees of realities. The constant evolution and refinement of our theories obviously gives the impression of profound qualitative changes, but this is to be put into perspective. In fact, our logical presuppositions vary little or do not change, as evidenced by the fact that it is almost impossible for us to spontaneously consider laws and behaviors that do not appeal to classical dualism (the law of the excluded middle in logic).

Our existence ultimately rests on an extremely limited set of rules forged from our daily experience so that we are able, as for example mathematicians and physicists do, to free ourselves from them only at the expense of difficult and tedious efforts of abstraction, efforts that on a social level are clearly reserved for a minority of the population (although a greater number of people are capable of it) and for which we must really evacuate our direct intuition to the benefit of a superior form of intuition in which we do not really understand much. 
If we observe the evolution of logic one finds how authentically qualitative changes are rare, as it took more than two millennia between Aristotle and G. Frege or between Aristotle and G. Boole for the logic to become truly modern. This logic as a product of human intelligence remains so deeply attached to that specific form of intelligence that it has developed only thanks to mathematicians whose intelligence appears precisely different, even super-natural, like a superintelligence. The current degree of abstraction of logic and, more generally, of mathematics and mathematical physics can no longer show advances in these disciplines as anything other than the productions of Alien individuals having succeeded in making a concept of intuition which completely escapes most individuals a material reality: in fact a cerebral faculty. Things happen not only as if the superintelligence escaped explanations - this is the case for some forms of strong artificial intelligence - but more radically still as if the phenomenology of superintelligence escaped all mental synthesis, in fact as it escaped phenomenology itself. We indeed perceive the effects of a new intelligence, but we do not grasp the rules, like young children in front of most phenomena or like adults who moved within a physical environment for tens of thousands of years without ever being able to faithfully reproduce most of its elements, at least before the invention of perspective. It is not uncommon to mention the Renaissance and the inventions of perspective and printing in the context of analysis of the current computational revolution, in general to bring the consequences of these 15th century inventions closer to the consequences of the invention of the computer. Nevertheless, if the inventions of perspective and printing have obviously marked the beginning of a new era, they may have even more embodied the end of a succession of previous eras and wanderings. Indeed, the perspective, prelude to a new vision of the world, was also the ordering - almost the final point - of a set of bodily-experienced perceptions never translated into spatial thought through scientifically articulated concepts for which both an absence of representations and rigorous geometric constructions were crippling.

There could be no real theory of space - everyone will understand that I consider geometric triangulation not as a theory of space but simply as a measurement - anterior to the invention of perspective for the simple reason that the foundations of an authentic understanding of space was lacking. How, in all rigor, theorize what one cannot even represent correctly? To better perceive what the absence of a general theory of space represented, it is enough to remember that for each region, country and tradition could be attached a specific representation stretching distances and planes, and deforming volumes and objects. With hindsight, we wonder by what miracle a seemingly unachievable peak of geometric intelligence appeared after long millennia is now within the reach of every child at the end of primary school. The task was authentically intractable, and the miracle consisted of a gradual evolution of our scientific methodology. If I come back to computation, although each interval of time of adaptation and time of adoption is always shorter, computation is socially comparable not to the invention of perspective as a "solution" - as the end of an era marked by the ordering of our perceptions - but largely to the appearance of what preceded, the Neolithic Period. Computation marks the entry into a new (post)history - 'the story of synthetic nature and Mendelized Campaign- entirely geared towards a process of 'cerebralization' for which the tools of construction and action on the world are given to us; just as they had been given to the greatest number by a few individuals or creative peoples, and obviously by parents to children, the tools allowing tillage, breeding, the crossing of rivers or the manufacture of boats. All that makes it possible to realize the dream of logical positivism - a logical (re)construction of the world and even more radically its logical emulation, i.e. its simulation - is now here, under our eyes and available. Nothing prevents us from making use of it, no more to travel in the physical and information space than to develop a new architecture.

If, as mentioned above, the space of superintelligence or superintelligent space is massively generated by computational procedures, many will tend to consider from the angle of causality that we understand this space and that we have circumscribed it. Nevertheless, contrary to classical and modern rationalist projects - the global Rationalist Project - whose origin can easily be found, the 
computationalist project of a superintelligent space is based on the strict impossibility of going back to the beginning of computational procedures for which most of the operations escape us (technically and theoretically speaking we will be able to run backward most of our computation, but again I do not consider here computation on a uniquely technical level). Behind each petabyte of data are hidden other petabytes, behind each algorithm, approximation and rounding operation are hidden thousands, millions or billions of other algorithms, approximations and rounding operations. Behind each step of a computation within a sequence certainly finite but that gigantic dimensions make infinite with regards to any human faculty, hide trillions of trillions of discrete states that act as trillions of trillions of partial causes. If, mathematically speaking, beginnings and ends do exist in finite-state machines, these concepts are among many other certainties no longer of any practical use, since they completely escape all perceptual capacities and all phenomenology, the latter being a transitional and now achieved stage in the history of theories of perception. Finally, one will now have to accept leaving things without explanations'. It might, perhaps, be 'characteristic of a superior culture' [freiedrich, 1996].

\section{References}

Bostrom, C. N. (2014). Superintelligence: Paths, Dangers, Strategies. Oxford University Press, Oxford.

Friedrich Nietzsche, 'It is necessary to leave things without explanations, it is the characteristic of a superior culture' 1996.

Hamming, R. W. (1980). The Unreasonable Effectiveness of Mathematics. The American Mathematical Monthly, Vol. 87, No. 2 .

Hamming, Richard Wesley. The Unreasonable Effectiveness of Mathematics, in. The American Mathematical Monthly, Vol. 87, No. 2., Feb. 1980.

Javier Seguí de la Riva and Ana Buenaventura's researches at the Computing Center of the University of Madrid (Centro de Calculos de la Universidad de Madrid), from 1968 to 1974, were recently presented at the first Orléans Architecture Bienale (Biennale d'Architecture d'Orléans; https://biennale-orleans.fr).

Kelly, Kevin. (2012, December 12). Better Than Human: Why Robots Will - And Must - Take Our Jobs. WIRED. Retrieved from https://www.wired.com/2012/12/ff-robots-will-take-our-jobs/" https://www.wired.com/2012/12/ffrobots-will-take-our-jobs.

Kurzweil, Ray. (2005). The Singularity Is Near: When Humans Transcend Biology. Penguin Books.

'La Superarchitettura è l'architettura della superproduzione, del superconsumo, della superinduzione al superconsumo, del supermarket, del superman e della benzina super.' As stated by the "Superarchitettura" exhibition (at Jolly 2 Gallery in Pistoia, Italy) manifesto presented at the opening, on December $4^{\text {th }}, 1966$.

Leary, Kyree. (2017, December 25). The Doctors of Tomorrow will be Supercomputers. NEOSCOPE. Retrieved from https://futurism.com/doctors-tomorrow-supercomputers.

Cite this article as: Morel P., "Towards an Artificial Architecture: About Superintelligent Space”, International Conference on the $4^{\text {th }}$ Game Set and Match (GSM4Q-2019), Doha, Qatar, 6-7 February 2019, https://doi. org/10.29117/gsm4q.2019.0026 